\title{
The effect of two types of induced-motion displays on perceived location of the induced target
}

\author{
JOSHUA H. BACON and AMIE GORDON \\ Tufts University, Medford, Massachusetts \\ and \\ PAUL H. SCHULMAN \\ State University of New York College of Technology, Utica, New York
}

\begin{abstract}
Using a pointing test, perceived location of a target seen in induced motion was evaluated under two display conditions. In one, a fixated, horizontally stationary spot was surrounded by a frame moving back and forth. As the frame moved to each side, its center shifted correspondingly with respect to the subject's objective median plane. In the second display, the surround was constructed so that as it moved back and forth, its center remained in virtual alignment with the objective median plane. Although both conditions produced a substantial induced-motion effect, only the former produced significant shifts in the target's perceived location. Furthermore, similar shifts were also obtained with a stationary, offcenter frame (Experiment 2). This suggests that the changes in perceived location obtained with the first induced-motion display were not derived from the induced motion per se, but, rather, from a frame effect produced when the surround moved to an off-center position. Implications for the relationship between perceived motion and position, as well as for two theories of induced motion, are discussed.
\end{abstract}

When a stationary, fixated target is surrounded by a moving frame, the target is often seen to travel in a direction opposite to that of the frame (Duncker, 1929). This induced-motion effect has been explained in terms of a perceptual resolution of conflicting information provided by subject- and object-relative motion cues (Wallach, 1965). Because target fixation produces no image displacement or eye movement pursuit, these subject-relative cues correctly signal the target's stationarity. However, the target may be perceived as moving if object-relative displacement alone is effective. This cue evokes a sense of motion through the changing distance or, in different terms, configurational change between a moving object and other objects in the visual array. Because of its relative nature, configurational change is, then, a cue only to the existence of motion within the array, but not to which object, in particular, is in motion. The fact that a stationary-target/movingsurround arrangement results in perceived motion of the target demonstrates dominance of configurational

Requests for reprints should be sent to Joshua Bacon, Department of Psychology, Tufts University, Medford, Massachusetts 02155. Amie Gordon's mailing address is the same as Joshua Bacon's; Paul H. Schulman's complete mailing address is: Department of Psychology, State University of New York College of Technology, Utica, New York 13502. change over the subject-relative cues when the two are in conflict.

One prediction that follows from a cue-selection interpretation of induced motion is that an observer may be able to accurately locate the stationary target (e.g., by pointing to its position) even, paradoxically, as it is seen to be moving. The basis of this prediction is the fact that the target's motion and position are derived from sources of information that, at least initially, are mutually exclusive. Perceived motion is a matter of configurational change, whereas perceived position is determined by a combined evaluation of three subject-relative position cues, namely, locus of retinal stimulation, eye position, and head position. If the subject-relative position cues remain independent and do not interact with the configurational information, they may, then, be accessible to guide accurate pointing to the target's position.

Using a procedure similar to one first devised by Farber (1979), we tested this prediction in a preliminary experiment by having subjects fixate a target seen in induced motion and simultaneously track its position with the unseen hand. The target, rather than being stationary or moving collinearly with the frame (as in Farber's study), moved vertically up and down in phase with the frame's lateral back-and-forth motion. Here, the target is perceived as moving obliquely, a result of its objective vertical motion and horizontal induced motion. 
Contrary to expectation, manual tracking was oblique, corresponding to the perceived and not the objective motion path.

Ostensibly, this result would seem to suggest that position cues are altered during induced motion. Still, as Bridgeman, Kirch, and Sperling (1981) have argued, manual tracking is potentially confounded as a test of perceived location. Consider, for example, the simplest situation, in which an observer is instructed to manually track the position of a fixated stationary target seen in induced motion. The target's position may be correctly given by subject-relative position cues and would ordinarily produce a stationary manual tracking response. However, the observer may be well aware of a logical inconsistency of seeing a target move back and forth while he/she points steadily to one location, and he may resolve the contradiction by moving his hand back and forth to mimic or match the perceived motion path. Thus, instead of measuring perceived location, manual tracking might actually be a secondary consequence of the perceived motion. Accordingly, in the first experiment reported below, instead of continuously tracking the target during induced motion, the subjects were instructed to wait until the frame and target were extinguished and then to point to the target's last seen position. Pointing responses to the target were obtained after it had reached either the top or the bottom of its motion path (and, correspondingly, the frame had reached either its leftmost or its rightmost lateral position). This endpoint test is a more accurate reflection of perceived location, since the subject is responding to the spot's momentary, immediate position without reference to its preceding motion path. Thus, if pointing responses to the target in its top or bottom position fall about a vertical line, it is clear that at the moment the target disappears, its position is correctly given by subject-relative position cues. If, however, the pointing responses correspond to endpoints of an oblique line matching the perceived motion path, we must necessarily conclude that perceived position is modified during induced motion.

\section{EXPERIMENT 1}

\section{Method}

Subjects. Ten undergraduates from Swarthmore College participated in this experiment; each was paid $\$ 2$ for the session.

Apparatus. The apparatus for presenting induced motion has been described in detail in a previous report (Wallach, Bacon, \& Schulman, 1978). In short, the surround consisted of a backilluminated translucent screen, $80 \mathrm{~cm}$ high and $50 \mathrm{~cm}$ wide, set in an aluminum frame. On the screen were 20 vertical lines, $.8 \mathrm{~mm}$ thick and evenly spaced at intervals of $2.5 \mathrm{~cm}$. The frame, attached to a variable-speed motor by a gear arrangement, moved back and forth along a track, traversing a horizontal distance of $8.7 \mathrm{~cm}$. The motion velocity changed in an approximately sinusoidal fashion at an average rate of $10 \mathrm{cycles} / \mathrm{min}$. A spot of light, $5 \mathrm{~mm}$ in diameter, was projected onto the screen by a device constructed from a plastic tube containing a light source, lens, and circular aperture. The projection tube was attached to the gear arrangement moving the frame in such a way that it could be made to tilt up and down in phase with the screen's motion. Specifically, as the screen moved from left to right, the spot moved in a vertical path from top to bottom. The extent of vertical motion was $15 \mathrm{~cm}$, so that the resultant of a fully effective horizontal induced motion and objective vertical motion would be a perceived oblique path going from lower left to upper right at an angle of $30 \mathrm{deg}$ from the vertical. Factors making induced motion less effective would result in a perceived oblique motion path that would be less than $30 \mathrm{deg}$, that is, closer to the vertical.

The display arrangement is illustrated in Figure 1. A large mirror was placed at a 45-deg angle to the subject's frontoparallel plane and at right angles to the frame display, causing a reflected image of the display to appear $40 \mathrm{~cm}$ in front. (Thus, as seen by the subject, the visual angle subtended by the frame amounted to $90 \times 64 \mathrm{deg}$, its lateral motion extent was $12.4 \mathrm{deg}$, and the target's vertical motion was $21.2 \mathrm{deg}$.) The real display was blocked from peripheral view by black cloth. Behind the mirror were two rulers, one above the other, extending horizontally in the same plane as the reflected image of the inducedmotion display. The distance between rulers was set at $15 \mathrm{~cm}$ so that the top horizontal edge of each corresponded to the vertically moving target's topmost and bottommost positions, respectively. The subject could then reach around the side of the mirror and, with the unseen hand, point along the ruler to the target's perceived position.

Procedure. The subject faced the mirror and observed three to four motion cycles. She or he was instructed to continuously fixate the spot and, when it disappeared, to point, with finger extended, along the appropriate ruler, to the position in which it was last seen. There were four pointing trials. In two, the spot and frame were extinguished after reaching the top and left positions, respectively, and in two, after reaching the bottom and right positions, respectively. Order of the four pointing trials was randomly counterbalanced for each subject.

In addition to the pointing test, a tilt-estimation test was given to measure directly the amount of induced motion. On the subject's right was a rod (visible against a dark background) that was attached perpendicularly to a horizontal shaft so that the rod could be set to any desired tilt. Each time a setting was made, a shielded $15-\mathrm{W}$ bulb was lit to illuminate the rod. After observing three to four motion cycles, the subject rotated the tilt rod to match the angle and direction of the perceived motion path. There were two trials in this condition, one with the tilt rod initially set to the vertical and one with it initially set to the horizontal. The pointing test was always given first.

\section{Results}

A pointing score was determined for each subject by computing the difference between the average hori-

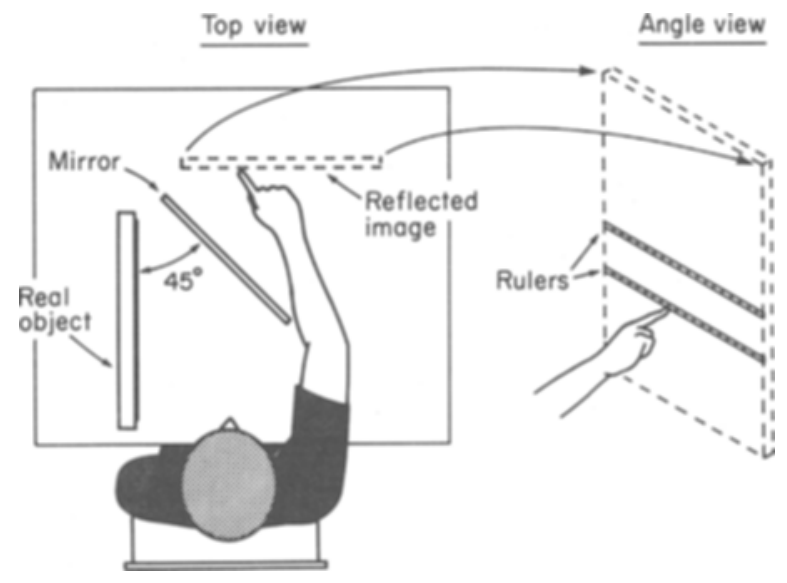

Figure 1. Schematic drawing of the display arrangement. 
zontal position of the two pointing responses macie to the spot where it disappeared at the top of its motion path and the average horizontal position of the two responses to the spot where it disappeared at the bottom of its motion path. The mean difference for the 10 subjects was $+6.75 \mathrm{~cm}(\mathrm{SD}=2.00)$. The positive sign indicates that pointing responses made to the bottom spot were shifted to the left of the responses made to the top spot. Thus, the pointing responses fell at the endpoints of a slanted line that corresponded to the endpoints of perceived and not objective motion.

The results of Experiment 1 demonstrate that the target's perceived momentary location is altered during induced motion. However, since the frame's horizontal displacement was $8.7 \mathrm{~cm}$, the horizontal difference in pointing responses should also have been $8.7 \mathrm{~cm}$. The obtained mean difference of $6.75 \mathrm{~cm}$ represents only $77 \%$ of a full induced-motion effect. The implication of this reduced effect may be made more evident by transforming the difference between horizontal pointing errors into actual slant angles. Since the position of the rulers fixes the vertical distance for the pointing responses to the top and bottom spots at $15 \mathrm{~cm}$, the slant angle corresponding to a $6.75-\mathrm{cm}$ horizontal shift may be determined by taking the arctangent of the ratio $6.75 / 15$. Computed in this way, the horizontal difference translates into a 24-deg slant angle, an underestimation of the full induced-motion effect, which would yield a slant angle of $30 \mathrm{deg}$. This slant angle is significantly smaller than the perceived slant angle of $40 \mathrm{deg}$ $(\mathrm{SD}=8.0)$ measured by the tilt estimate test [tcorr(14) $=4.00, p<.01]$. Since the magnitude of the obtained tilt estimates corresponded to the full predicted inducedmotion effect, it is apparent that induced motion was complete but only partially measured by the endpoint test. Whether or not this underestimation is a consistent characteristic of the test will be considered later in the paper.

\section{EXPERIMENT 2}

The endpoint test results show that the conditions producing induced motion also produce changes in perceived location. However, the very fact that a pointing effect can be measured after the frame has reached a fixed position raises the question of whether phenomenal experience of induced motion is even a necessary component for producing changes in perceived location. For example, the frame's motion even without a target (and hence, without the experience of induced motion) or simply a stationary frame that is off-center relative to the observer's median plane may be sufficient to produce changes in perceived location. Accordingly, in Experiment 2, the endpoint test was administered following three viewing conditions: after induced motion, after a back-and-forth motion of the frame in the absence of the spot, and after the presentation of a stationary, off-center frame.
Method

Subjects. Eight undergraduates at Tufts University served as subjects; each was paid $\$ 2$ for the session.

Apparatus and Procedure. A different display was used in this experiment. The visual patterns and sequence of motions corresponding to the different experimental conditions were programmed on a PDP-8/e computer and displayed on a 505 fast-phosphor Tektronix oscilloscope. Each condition was taped on a Panasonic NV 8030 video recorder using a low-light sensitive camera (Panasonic WV-1350A) and played back on a large video monitor $(49 \times 38 \mathrm{~cm})$. The brightness and contrast control on the monitor were set to a sufficiently low level so that only the movement patterns would be visible. The arrangement for observing the display was similar to that of the previous experiment. A mirror was placed at a $45-\mathrm{deg}$ angle to the video monitor, and the subject, sitting at right angles to the monitor and facing the mirror, saw the reflected image behind the mirror at a distance of $40 \mathrm{~cm}$. By reaching around the mirror, the subject could point with the unseen hand at the reflected image of the spot as the conditions required. However, in contrast to the earlier experiment, the subject was allowed the freedom to point in both the horizontal and the vertical dimensions. The two-ruler arrangement behind the mirror was eliminated and replaced with a large board affixed at right angles to the surface of the table so that it was in the same plane as the image of the induced motion display. A paper was attached to the board, and the pointing responses were recorded directly on this paper. A thin pointer was attached to the end of the subject's index finger so that a precise mark could be made of each response.

Each subject was presented with three display conditions given in randomly counterbalanced order. In the induced-motion condition, a frame consisting of two $5-\mathrm{mm}$ thick vertical lines, $27 \mathrm{~cm}$ high and separated by a distance of $19.5 \mathrm{~cm}$ (thus subtending a visual angle of $37 \times 27 \mathrm{deg}$ ), moved back and forth a horizontal distance of $11 \mathrm{~cm}$ (15.4 deg of visual angle) at a constant velocity of $4.5 \mathrm{~cm} / \mathrm{sec}$. Within the frame was a spot, $5 \mathrm{~mm}$ in diameter, that moved vertically in phase with the horizontal motion of the frame at an equal constant velocity of $4.5 \mathrm{~cm} / \mathrm{sec}$. The extent of the vertical motion was also $11 \mathrm{~cm}$, so that the result of a fully effective horizontal induced-motion component and objective vertical motion component would be a perceived slanted motion path of $45 \mathrm{deg}$. The subject observed the motion display through three to four cycles and, when the frame and spot disappeared, pointed to the spot's last seen position. There were four pointing trials, two with the frame in the right position and the spot at the bottom of its motion path and two with the frame in the left position and the spot at the top of its motion path. The order of the four trials was randomly counterbalanced for each subject.

In the induced-motion control condition, the spot was eliminated during the frame's back-and-forth motion. At the very end of the last cycle, when the frame reached one of the two extreme lateral positions, the spot appeared momentarily and then disappeared with the frame. Four trials were administered. In two, when the frame reached the left position, the spot appeared in the location that would have been the top of its motion path (as given in the induced-motion condition), and in two, when the frame reached the right position, the spot appeared in the location that would have been at the bottom of its motion path. The four trials were presented in randomly counterbalanced order. Subjects were not given any specific fixation instruction while the frame moved back and forth, but they were told that, after the spot appeared and disappeared (along with the frame), to point to its last seen position. In this condition, the effect of the motion of the frame independent of induced motion could be tested.

In the stationary off-center frame condition, the frame and spot appeared for $1 \mathrm{sec}$ as a static display and then disappeared. Once again, there were four trials, two with the frame to the left and the spot in the position corresponding to the top of its motion path (as given in the induced motion condition) and two 
with the frame to the right and the spot in the position corresponding to the bottom of its motion path. The order of the four presentations was randomly counterbalanced.

\section{Results}

The four pointing responses for each condition were analyzed in the following manner. An average of the two pointing responses to the spot in its top position and an average of the two pointing responses to the spot in its bottom location were computed. Since pointing was not restricted, the computed location was determined by the average pointing error in both the horizontal and vertical dimensions. A line was then drawn to connect the average pointing response to the top spot and the average response to the bottom spot. The slant angle of this line represents the degree of shift in the target's perceived position. A zero angle would correspond to the objective locations of the top and bottom spots, whereas a 45-deg slant would represent a full change in perceived position commensurate with the distance between the two extreme positions of the frame.

The mean slant angles obtained in the inducedmotion, induced-motion control, and stationary offcenter frame conditions were 32.67 (SD = 14.27), 37.13 $(S D=14.05)$, and $30.0(S D=9.49)$ deg, respectively. $A$ one-way analysis of variance for repeated measures showed that the difference between the means was not significant $[F(2,14)=1.48, p>.05]$, indicating that moving and stationary off-center frames produce similar changes in the target's perceived location. It may be noted that as in the endpoint test of Experiment 2, the shift in perceived location as measured by the pointing response is only a proportion of the frame's two extreme positions. For example, in the induced-motion condition, the perceived oblique motion path should be $45 \mathrm{deg}$, but the endpoint test yields a slant angle of only $33 \mathrm{deg}$. However, since a tilt-estimation test was not included in this experiment, there is no direct measure of the amount of induced motion. It is, thus, possible that factors in the new display, such as screen size, constant velocity of motion (in contrast to the sinusoidal motion of the earlier experiments), and so on, may have made this induced motion less effective. The induced motion condition was therefore repeated with 12 new subjects using both the tilt-estimation test and the endpoint test, presented in randomly counterbalanced order. The mean for the former was $39.7 \mathrm{deg}$ $(S D=6.53)$, and that for the latter was $26.33 \mathrm{deg}$ $(\mathrm{SD}=13.55)$. The difference between these means is highly significant (tcorr $=3.24, \mathrm{p}<.01$ ), indicating that the endpoint test consistently measures only a proportion of the full induced-motion effect.

\section{EXPERIMENT 3}

The first two experiments show that a moving surround and a stationary off-center surround produce similar shifts in a target's perceived location. Within the context of a selection-of-cues interpretation of induced motion, the pointing effect obtained with a moving frame could be explained in two ways. One could maintain the original premise that motion and position are independent noninteractive processes and the stimulus cues causing or affecting one do not influence the other. In this case, changes in the target's perceived location obtained with a stationary off-center or moving surround could be attributed to the effect these two conditions have on the apparent straightahead. In particular, the apparent straightahead shifts toward the middle of a stationary off-center frame (Roelofs, 1935) and shifts continuously toward the center of a moving frame (Brosgole, 1968). Consider now a situation in which a frame has moved to the right of the objective median plane or is presented in that position as a stationary display. In accordance with the Brosgole and Roelofs effects, respectively, apparent straightahead will also shift to the right. By inference, a target in the objective median plane will appear to the left of apparent straightahead and pointing errors would be to the left of the target's true position, as our results indeed showed.

Alternatively, the premise that motion and position are noninteractive might be incorrect and perceived motion could in some way force a reevaluation of position cues to bring perceived motion of the target and its perceived location into correspondence. According to this explanation, the shift in perceived location obtained with a moving or stationary off-center surround would be attributed to separate psychological processes, the former to an effect produced by induced motion and the latter to a Roelofs (1935) effect.

In Experiment 3, a test between the two explanations was made by presenting a display in which a surround moved back and forth but was constructed so that its center always remained straight ahead in the objective median plane. According to the first explanation, eliminating the stimulus that produces changes in the apparent straightahead (i.e., an off-center frame) should allow the target's position to be accurately located. However, if such a display produces induced motion, then, according to the second interpretation, perceived location should be modified by the perceived motion and should result in a significant pointing effect.

\section{Method}

Subjects. Sixteen undergraduate students from Tufts University served as subjects. For participating, subjects received either $\$ 3$ or credit for fulfilling a requirement in an introductory psychology course. Two of these subjects were subsequently eliminated from analysis because of scores more than 2 standard deviations from the mean.

Apparatus and Procedure. Two different induced-motion displays were used. One, the aligned-center pattern, consisted of 15 vertical lines, $30 \mathrm{~cm}$ high and evenly spaced at intervals of $2.5 \mathrm{~cm}$. The total width of the surround was therefore $45 \mathrm{~cm}$. The pattern moved back and forth in reciprocating fashion with a vertical moving target, but the motion of the lines was seen as if they were behind an imaginary window $47.5 \mathrm{~cm}$ wide (43.2 deg of visual angle). When the pattern moved the width 
of the spatial period (i.e., $2.5 \mathrm{~cm}$ ), one line disappeared on the right side of the display and another appeared on the left side. Thus, the center of the display (i.e., the middle of the "window') was always in virtual alignment with the objective median plane. The actual extent of horizontal motion, defined in terms of the displacement of a particular line, was $23 \mathrm{~cm}$. The target's vertical motion was $19 \mathrm{~cm}$, so that a fully effective induced effect would correspond to a tilt angle of $40 \mathrm{deg}$.

The second display, the moving-center pattern, consisted of 10 vertical lines moving back and forth in reciprocating motion with a vertically moving target. In this case, the lines constituted a frame whose center moved back and forth with respect to the objective median plane and, so, was analogous to the displays of Experiments 1 and 2.

The arrangement for presenting the displays was identical to that in Experiment 2, with the exception that the viewing distance was $60 \mathrm{~cm}$.

Four pointing measures were obtained for each display condition. After the pattern moved back and forth through three to four cycles, both the target and the surround disappeared, and the subject pointed to the target's last seen position. In two of the trials, the pattern disappeared when the target reached the top position of its motion path, and in two, the pattern disappeared after the target reached the bottom of its motion path. The procedure was then repeated for the other display condition. The order of presentation of the two conditions was randomly counterbalanced across subjects. After the pointing trials, each subject also made a tilt estimate for both displays. Two measures were made for each display, with order of presentation the same as it had been for that subject in the pointing test.

\section{Results}

The four pointing responses for each condition were analyzed as in Experiment 2. For the moving-center display, the mean pointing effect corresponded to a slant angle of $26.2 \mathrm{deg}(\mathrm{SD}=11.16)$ and the mean tilt estimate was $39.1 \mathrm{deg}(\mathrm{SD}=6.93)$. These results effectively replicate the pointing effect and tilt estimates obtained for the moving-center displays of Experiments 1 and 2.

For the aligned-center display, the mean pointing effect corresponded to a slant angle of $5.5 \mathrm{deg}(\mathrm{SD}=$ 10.09). This effect was not reliably different from the null effect (i.e., a vertical slant angle) $[t(13)=2.04$, $\mathrm{p}>.05]$ and was significantly different from the pointing mean obtained with the moving-center display $[\mathrm{t}(13)=8.60, \mathrm{p}<.001]$. Thus, only the moving-center pattern produced reliable changes in perception of the target's location.

The mean tilt estimate obtained for the aligned-center display was $31.4 \mathrm{deg}(\mathrm{SD}=10.95)$. This mean was significantly different from the null effect $[t(13)=10.72$, $\mathrm{p}<.001]$, but it was also significantly smaller (by $7.7 \mathrm{deg}$ ) than the tilt estimate obtained with the movingcenter display $[\mathrm{t}(13)=3.06, \mathrm{p}<.02]$.

\section{GENERAL DISCUSSION}

Experiment 3 clarifies two issues concerning induced motion. The results showing a pointing effect only with a moving-center display but not with an aligned-center pattern indicate that the target's changing perceived location is not a consequence of induced motion but is specifically due to the presence of an off-center frame. When this factor is eliminated from the display, subjectrelative position cues provide accurate information about the target's position, which is unaffected by the perceived motion path. This result is consistent with a study by Hansen and Skavenski (1977), who used a motion situation different from ours but also found perceived position and perceived motion to be independent noninteractive processes. The display consisted of two dots moving back and forth in reciprocating fashion, one along the vertical leg and one along the horizontal leg of a right angle. In this case, the dots are seen as moving directly toward and away from each other (along the hypotenuse), and yet the position of a dot is accurately located (e.g., by striking it with a hammer).

Interestingly, Bridgeman et al. (1981) recently reported a study in which they used an induced-motion display similar to our moving-center condition and found a pointing effect that, although highly significant, was relatively small in comparison with the target's objective egocentric position. Although we have no definitive explanation for the discrepancy between the two studies, there are several factors that may account for the reduced pointing effect reported by Bridgeman et al. The induced-motion effect per se was only $2.5 \mathrm{deg}$ of visual angle, less than $20 \%$ of the potential effect of $14 \mathrm{deg}$; in all of the analogous conditions (movingcenter displays) of the present experiments, induced motion was virtually complete. This may be due to the fact that Bridgeman et al. used a stationary target, a situation that, in our experience, has generally led to a smaller and substantially more variable effect (see, e.g., Wallach et al., 1978). Presumably, whether the eyes fixate on the stationary target or follow the moving background, there are additional subject-relative cues (e.g., stationary eyes in fixed binocular convergence or eye movement pursuit, and image displacement of the target when, respectively, the target or background is fixated) that override the normally prepotent objectrelative information. In a similar fashion, these additional cues may result in an off-center frame's displacement being correctly perceived, and in consequence, the target's position would be accurately registered. When these additional subject-relative cues are eliminated by having the subject track a vertically moving target, as in our experiments, the frame's displacement may not be processed as such, but, rather, as always being straight ahead. The perceived position of the target would then necessarily change in the direction indicated by our pointing data.

A second factor in the Bridgeman et al. (1981) study is that only a few subjects were tested, but each was presented with 120 trials for any given condition. This raises the possibility that their pointing responses reflected primarily an adaptation to the off-center frame effect. Thus, the frame's potential effectiveness in producing perceived position shifts in the target may have been clearly evident in early trials but have decreased 
through repeated exposure. The effect measured over all trials would, then, be relatively small. Importantly, this adaptation need not (and, apparently, did not) occur in the perceived induced motion, since, as our Experiment 3 shows (and as we will discuss more fully below), perceived induced motion is independent of the presence of an off-center frame. Thus, while we agree with the principal conclusion of Bridgeman et al. that the pointing response is independent of perceived motion, our results suggest this to be the case only when offcenter frame effects have been eliminated. When the background is a moving-center arrangement, however, perceived position does change substantially in accordance with the off-center frame. ${ }^{1}$

Experiment 3 also resolves, in part, a theoretical debate concerning the underlying cause of induced motion. As discussed earlier, the Brosgole (1968) and Roelofs (1935) effects indicate a tendency of apparent straightahead to remain aligned with the center of a moving or stationary off-center frame, respectively. Brosgole proposed that induced motion is, in fact, not at all a matter of configurational change, as we have assumed throughout, but, rather, is derived from these frame-produced shifts in perceived location. Thus, if the center of a moving frame always appears to be straight ahead, then, by inference, an objectively stationary target would appear to move back and forth in relation to this shifting egocentric reference. However, our results showing a substantial induced motion with an aligned-center display provide conclusive evidence that configurational change alone, without an off-center frame effect, is sufficient to produce perceived motion of the target. ${ }^{2}$ Of course, the pointing effect obtained with moving-center displays shows that shifts in the perceived location of a target do occur when an offcenter frame is involved. Nevertheless, contrary to Brosgole's contention, the cause-and-effect relationship between these shifts and induced motion, even for that particular type of display, cannot be decisively determined. For example, there may be two different types of induced motion, one that results from configurational cues (aligned-center display) and one, a Brosgole type, that results from perceived location shifts produced by the off-center frame effect (moving-center display). Accordingly, our assertion that perceived motion and perceived location are independent would be limited to those conditions, such as an aligned-center pattern or a two-dot arrangement, that do not contain an off-center frame. Alternatively, perceived motion and perceived position may always be independent, noninteractive processes. Induced motion, then, for both alignedcenter and moving-center displays could be attributed to the effectiveness of configurational change. However, for the latter display, as the frame moves off-center, it produces concomitant change in the target's perceived location that, coincidentally, corresponds to the direction of perceived motion.

Both approaches can obviously explain the effects obtained with moving-center displays. However, the consistant quantitative discrepancy we have noted between the pointing responses and amount of induced motion measured with a tilt test is suggestive evidence in favor of the latter interpretation, which postulates independent motion and position processes for both types of displays. If induced motion in a moving-center display is caused by an off-center frame effect, then one would expect the change in perceived location and amount of induced motion to be identical, which we have shown is not the case, or, at the very least, highly correlated. In fact, a correlation coefficient computed between the pointing and tilt-estimation tests for the moving-center display conditions across all three experiments was negligible $(r=.04)$. Thus, the tests do not agree either quantitatively or qualitatively. On the other hand, if motion and position are derived from independent cues, there is no reason to expect the pointing and tilt-estimate responses to correspond, since configurational change responsible for perceived motion could be fully effective, whereas the off-center frame effect, which produces perceived position change, is not.

In conclusion, while both a moving-center display and an aligned-center display produce substantial induced motion, perceived location shifts in the target are obtained only with the former. The fact that the pointing effect is consistently smaller and is uncorrelated with the amount of induced motion, together with the stationary off-center frame effect, suggests that motion and position in all cases are derived from independent noninteractive processes.

\section{REFERENCES}

Bridgeman, B., Kirch, M., \& Sperling, A. Segregation of cognitive and motor aspects of visual function using induced motion. Perception \& Psychophysics, 1981, 29, 336-342.

Brosgole, L. Analysis of induced motion. Acta Psychologia, $1968,28,1-44$.

DAY, R. H., \& Dickinson, R. G. Absence of color-selectivity in Duncker-type induced visual movement. Perception \& Psychophysics, 1977, 22, 313-320.

Duncker, K. Uber induzierte bewegung. Psychologische Forschung, 1929, 12, 180-259.

Farber, J. M. Manual tracking of object induced motion. Investigative Ophthalmology, 1979(S), 3.

Hansen, R., \& Skavenski, A. Accuracy of eye position information for motor control. Vision Research, 1977, 17, 919-926.

Over, R., \& Lovegrove, W. Color selectivity in simultaneous motion contrast. Perception \& Psychophysics, 1973, 14, 445448.

Rozlors, C. O. Optische lokalisation. Archiv fur A ugenheilkunde, $1935,109,395-415$.

WALlACH, H. Visual perception of motion. In G. Kepes (Ed.), The nature and the art of motion. New York: Brasiller, 1965.

Wallach, H., Bacon, J. H., \& Schulman, P. Adaptation in motion perception: Alteration of induced motion. Perception \& Psychophysics, 1978, 24, 509-514.

Wong, E., \& MAck, A. Saccadic programming and perceived location. Acta Psychologica, 1981, 48, 123-131.

\section{NOTES}

1. However, a recent study by Wong and Mack (1981) suggests that saccades are driven directly from the untransformed retinal signal even in the presence of an off-center frame. 
2. An alternative explanation of our aligned-center results is, however, possible. The target motion seen in an aligned-center display may be an example of simultaneous motion contrast (SMC), an effect attributed to center-surround interaction in a retinal velocity-detecting unit (e.g., Over \& Lovegrove, 1973). Evidence suggesting that $\mathrm{SMC}$ may be derived from peripheral activity (and is, therefore, presumably different from the centrally determined induced motion) comes from a study showing that when a moving pattern within a stationary aperture is presented to one eye and a stationary target to the other eye, induction of the target does not occur (Day \& Dickinson, 1977). With the aperture removed (thus producing a display similar to our moving-center condition), dichoptic induced motion is easily observed. Nevertheless, as Day and Dickinson emphasize, it is not known whether this difference in dichoptic effects with the two conditions indeed reflects two fundamentally different types of motion or, instead, is an artifact of the consequences of inappropriate binocular fusion. In fact, we have found that dichoptic induction can be readily seen with our aligned-center display when binocular fusion is controlled, and we are, therefore, inclined to believe that the induction obtained with the alignedcenter display is not a matter of SMC.

(Manuscript received December 22, 1981; revision accepted for publication July 12,1982 .) 